\title{
Letter on a Prospective Study of the Efficacy of Cell-Assisted Lipotransfer with Stromal Vascular Fraction to Correct Contour Deformities of the Autologous Reconstructed Breast
}

\author{
Ping Cao ${ }^{1} \cdot \mathrm{Xi} \mathrm{Xiang}^{1} \cdot$ Chunying Chen ${ }^{2}$
}

Received: 9 October 2020/ Accepted: 14 October 2020/Published online: 3 November 2020

(C) Springer Science+Business Media, LLC, part of Springer Nature and International Society of Aesthetic Plastic Surgery 2020

Level of Evidence $V$ This journal requires that authors assign a level of evidence to each article. For a full description of these Evidence-Based Medicine ratings, please refer to the Table of Contents or the online Instructions to Authors www.springer.com/00266.

Sir,

We read with great interest the article entitled "A Prospective Study of the Efficacy of Cell-Assisted Lipotransfer with Stromal Vascular Fraction to Correct Contour Deformities of the Autologous Reconstructed Breast" by Jeon et al. [1] in Aesthetic Plastic Surgery. In this article, the authors conducted a 12-month prospective study on the efficacy of stromal vascular fraction (SVF)-assisted fat grafting on the volume deficit following autologous breast reconstruction using a transverse rectus abdominis myocutaneous flap or latissimus dorsi flap after total mastectomy. This study showed that the cell-assisted lipotransfer (CAL) with SVF is superior to CAL alone in terms of volume augmentation and patient satisfaction. The authors' work provided a new idea for the treatment of contour deformities after flap surgery and will encourage more exploration from the scientific community.

In this study, the total sample size was 20. The conclusion obtained resulting from the small sample size is

Chunying Chen

chunyingchen126@126.com

1 Tianyou Hospital, Wuhan University of Science and Technology, No. 9 DingZi Bridge TuJiaLing, WuChang District, Wuhan 430064, People's Republic of China

2 Central South Hospital of Wuhan University, No.169 Donghu Road Wuchang District, Wuhan City, Hubei Province, People's Republic of China less convincing. We suggest that the sample size should be increased to reduce bias.

For the SVF isolation, the authors used collagenase. After transplantation, residual collagenase has a potential risk of alterant microenvironment in the recipient area. Unlike a single-cell SVF [cellular SVF (cSVF)] isolated by collagenase, the nonenzymatic isolation procedures, called mechanical dissociation, result in tissue SVF (tSVF). [2] The tSVF may be safer and more effective than cSVF due to the avoidance of collagenase and with intact cell-cell communications. Thus, the use of tSVF for volume augmentation may have more potential for regenerative purposes.

\section{Compliance with Ethical Standards}

Conflict of Interest The authors declare that they have no conflicts of interest to disclose.

Human and Animals Rights This article does not contain any studies with human participants or animals performed by any of the authors.

Informed Consent For this type of study informed consent is not required.

\section{References}

1. Jeon HJ, Choi DH, Lee JH et al. (2020) A prospective study of the efficacy of cell-assisted lipotransfer with stromal vascular fraction to correct contour deformities of the autologous reconstructed breast. Aesth Plast Surg 1-11(epub ahead of print)

2. van Dongen JA, Tuin AJ, Spiekman M et al (2018) Comparison of intraoperative procedures for isolation of clinical grade stromal vascular fraction for regenerative purposes: a systematic review. J Tissue Eng Regen Med 12(1):e261-e274

Publisher's Note Springer Nature remains neutral with regard to jurisdictional claims in published maps and institutional affiliations. 